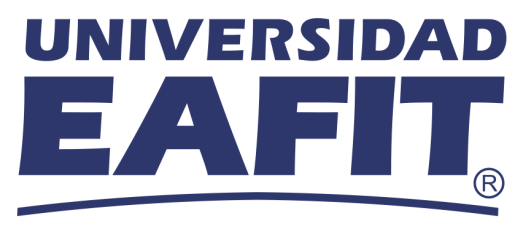

\title{
Solid Domestic Waste classification using Image Processing and Machine Learning
}

\author{
Daniel Otero Gómez 1
}

\author{
Advisors: \\ Mauricio Toro Bermúdez 2 \\ William Hoyos Morales ${ }^{3}$
}

\author{
Research practice I \\ Research proposal \\ Mathematical Engineering \\ Department of Mathematical Sciences \\ School of Sciences \\ Universidad EAFIT
}

January 2021

\footnotetext{
${ }^{1}$ Mathematical Engineering Student at Universidad EAFIT (CvLAC: Daniel Otero Gómez)

${ }^{2}$ IT and Systems Department professor at Universidad EAFIT mtorobe@eafit.edu.co

${ }^{3}$ Doctoral Student at Universidad EAFIT whoyos@correo.unicordoba.edu.co
} 


\begin{abstract}
This research concentrates on a bounded version of the waste image classification problem. It focuses on determining the more useful approach when working with two kinds of feature vectors, one construed using pixel values and the second construed from a Bag of Features (BoF). Several image processing techniques such as object centering, pixel value re scaling and edge filtering are applied. Logistic Regression, K Nearest Neighbors, and Support Vector Machines are used as classification algorithms. Experiments demonstrate that object centering significantly improves models' performance when working with pixel values. Moreover, it is determined that by generating sufficiently simple data relations the BoF approach achieves superior overall results. The Support Vector Machine achieved a 0.9 AUC Score and 0.84 accuracy score.
\end{abstract}

Keywords: Machine Learning, Image Classification, Image Processing, Bag of Features, Support Vector Machine

\title{
1 Introduction
}

Waste treatment has become a heavily relevant topic due to the important social, environmental, and economical consequences that carry such activity. Inappropriate debris management may cause several natural damages such as soil, water, and air pollution, in addition to pubic health deterioration (Aguilar-Virgen et al. , 2010). Research indicates that since 19508.3 billion tons of solely plastic were produced. Moreover, 6.3 billion tons were disposed as debris, from which $9 \%$ were recycled, $12 \%$ were incinerated, and $73 \%$ were located in landfills (Geyer et al., 2017). This is highly alarming due to the fact that the expected time of soil destruction of non-organic wastes is quite long. For instance, glass lasts one million years to be completely destroyed, and plastic, depending of the type, may vary from 58 to 1200 years (Chamas et al., 2020) (Mohajerani et al. 2017). Appropriate waste management must not be underestimated.

Solid Domestic Waste (SDW) corresponds to solid elements of day-to-day use that are discarded by households and several commercial and community centers, such as malls, workplaces, universities, etc. Plastic and glass bottles, food containers, soda cans, paper, or cardboard are just a few of the garbage that can be labeled as a SDW. An inter-continental study performed in 22 different countries determined that, in average, households contribute $55-88 \%$ of municipal solid wastes and that market and comercial areas contribute a 10-30\% (Abdel-Shafy \& Mansour, 2018). Since SDW is produced at such a high density and intensity it is crucial that a correct treatment is given to it.

Waste classification plays a major role in the waste treatment cycle since it allows to segregate wastes in order to prevent environmental damages and collect recyclable wastes. For example, useful SDW treatment allows that local community rubbish is recycled or reused in other processes (Aguilar-Virgen et al. , 2010). Despite the importance of such practices there is plenty of things to be done about waste management. Most recycling systems rely on human force for debris decomposition, practices that are both expensive and time consuming. Furthermore, citizenship has failed to acquire proper environmental practices. According to a study made in Colombia in 2020, 78\% of households do not recycle their wastes (Semana, 2020) (Andesco, 2020).

Such issues have motivated the scientific community to work on technological developments that contribute to the solution of these problems. Some of the main efforts focus on automatizing waste classification at different levels. Research scopes can vary from the development of mobile 
apps, embedding classification models in cameras located in trash cans, to the automatization of industrial and mass waste treatment (Toğaçar et al., 2019) (Mittal et al., 2016) (Ozkan et al., 2014). Additionally, garbage bin level detection has also become popular topic among the scientific community (Hannan et al., 2012) (Arebey et al., 2011).

This research is going to focus on the development of a classification model for solid domestic waste images using processing techniques and classical machine learning algorithms. This article compiles the results of an exploratory study on the implementation of two different methods of feature extraction for the development a bounded version of the waste classification problem. The present project will serve as a basis for the development of the problem at its full scope.

The motive of conducting this research is to gain understanding of classical techniques implemented in Computer Vision and Machine Learning. Adopting modern and more advanced methods without comprehending the basis from which this algorithms were developed generally conducts to committing errors due to the lack of understanding of the area. The knowledge acquired is planned to be applied in further stages of the project in order to properly to take advantage of state of art algorithms and methodologies.

The remaining part of the article will be structured as it follows. Section 2 contains a literature review of research papers related to the topic. The review is mainly focused on the two main characteristics of pattern recognition problems, feature extraction and classification algorithms. The implemented methods and algorithms along the data set are explained in Section 3. In Section 4, a compilation of the experimentation and results can be found. Finally, Section 5 contains the conclusions and future work.

\section{Related Work}

Automated systems for waste separation using image processing and artificial intelligence have been a topic widely studied in recent years. A few articles describe the pipeline that the images go through to make the classification. In Liu et al. (2018) and in Aslan et al. (2020) Speed Up Robust Features (SURF) is used in order to perform the feature extraction. Liu et al. (2018) stated that "... SURF has an excellent effect on image recognition and classification with fast processing speed". SURF extracts unique large dimension descriptors of the image, which makes the method suitable for image matching. Feeding this amount of information to classification algorithms often decreases the models' accuracy, which is why is recommended to apply a technique for dimension reduction to the descriptor. The Bag-of-Words (BoW) method is implemented after SURF has generated the descriptors. The operations performed by BoW are based on the idea that relying on the existence of a dictionary of words, each element of the vector corresponds to a word in the dictionary, thus, each image can be described by an $\mathrm{N}$-dimensional vector of words where the order of the words is negligible. Word mapping is accomplished through a K-Means clustering that maps N objects into K clusters. Finally, a Multi-Class Support Vector Machine (SVM) is used to perform the classification, achieving an average of $83.38 \%$ within five categories (Liu et al., 2018).

In Geyer et al. (2017) a different approach is taken. Geyer et al. (2017) research focuses on the classification of three different types of plastic bottles using a manually construed data set 
of 90 images properly balanced by class. The feature vector is build by flattening the image matrix and performing classification with a majority voting scheme using a Support Vector Machine. Principal Components Analysis, Kernel PCA, Fishers Linear Discriminant Analysis, Singular Value Decomposition, and Laplacian Eigmaps are applied separately to the feature space. Afterwards, the algorithm is separately fed with each input and the most voted option is the one chosen. The research achieved an $88 \%$ of overall accuracy. Furthermore, Kumar \& Bhatia (2014) make a review of the different methods that are currently used for feature extraction.

Both Deep Learning (DL) and Machine Learning (ML) approaches have been implemented by several authors, some of them achieving promising results regarding image classification. In Sakr et al. (2016) DL and ML approaches are compared. A Convolutional Neural Network (CNN) and an SVM are utilized to perform the comparison. Neural Networks architecture allows the model to automatically learn most features of the image. This makes these algorithms particularly powerful for image recognition. The SVM algorithm has gained a lot of attention recently, demonstrating to be useful in multiple application fields such as medicine, remote sensing, and environmental sciences (Camlica et al., 2015) (Liu et al., 2017) (Liu et al. 2018). The SVM was implemented along a Bag-of-Features (BoF) techniques. Such methods are similar to BoW, however, it differs from it by the fact that the features are described after running a kernel over the image matrix. The data set originally contained 2000 images, however, it was widened using label-preserving transformations, ending up with 6000 images. To conclude, the CNN achieved an $83 \%$ accuracy, while the SVM achieved $94.8 \%$.

On the contrary, Satvilkar (2018) performed a comparative review of the SVM, Random Forest, K Nearest Neighbors, eXtreme Gradient Boost, and a CNN for the classification of 2390 image data set of five different waste classes. Validation of sampling adequacy techniques such as the Kaisen Meyer Olkin Test were performed in order to withdrew non-adequate images. PCA was applied to the feature vector as well. The classifiers performance was evaluated using the accuracy. CNN labeled correctly $89 \%$ of the data, whilst, the other algorithms accuracy ranged between 50 and 70 percent of accuracy. Despite the results, classical algorithms performance must not be underestimated. The results merely indicate that more powerful preprocessing techniques must be considered.

To conclude, it is clear that the SVM receives particular attention in image classification problems. It is common to approach the problem from a multi-class classification perspective, thus, it is important to address the models performance in such situations. In Tomar \& Agarwal (2015) comparison of multi-class classification methods is performed in order to determine which method induces a better performance for SVM multi-class problems, finding that the DAG MLSTSVM classifier provides a better performance.

\section{Methodology}

\subsection{The data set}

The data set was extracted from a public repository that can be found in here. It contains 2532 images of six different classes: cardboard, glass, metal, paper, plastic and trash. The long-term goal of the research is to develop a model capable of classifying multiple types of waste into several categories, however, successfully attaining such an objective requires a more extended period time. 
Hence, the scope of the first phase of the project, an exploratory one, was bounded to consider performing binary classification using cardboard an metal classes. Each label is composed of 403 and 410 RGB images, respectively, of $384 \times 512$ in .jpg format. A sample of the images is shown below.

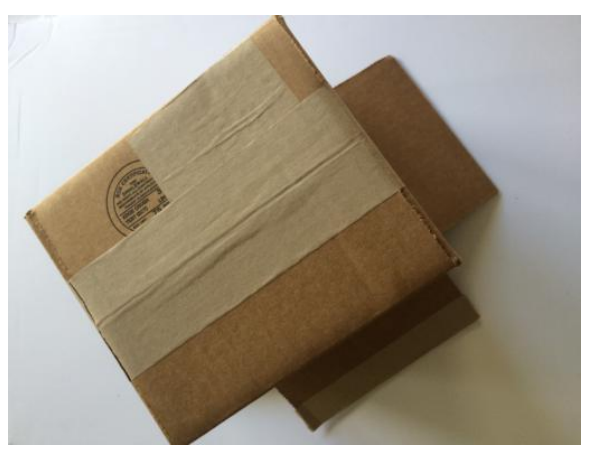

Figure 1: Cardboard image sample.

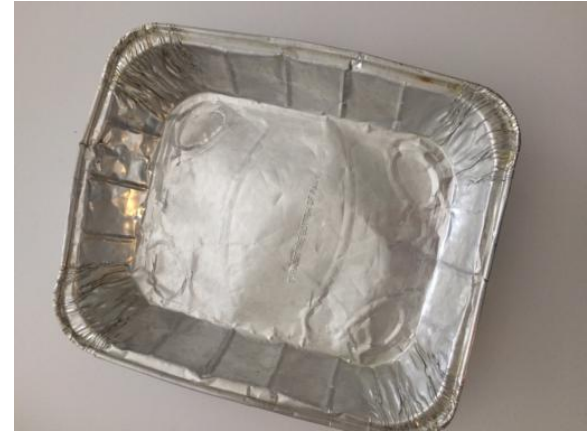

Figure 2: Metal image sample.

\subsection{Feature extraction}

Feature extraction is a crucial part of every image classification problem. It's main objective is to represent the image through a feature vector that best summarizes the main characteristics of an image. This paper considers two different approaches, working with the image pixels and a Bag of Features $(\mathrm{BoF})$.

\subsubsection{Image pixels}

Images are represented as matrices, thus, the traditional approach when considering pixel values as a feature vector is to reshape the matrix into a single vector. This process is called flattening and it can be performed across any of the axes of the matrix. The main idea is to desegregate the matrix by each row or column, depending on the chosen axis, and concatenate the vectors until the matrix is expressed as a single vector. This process is illustrated in the figure shown below.

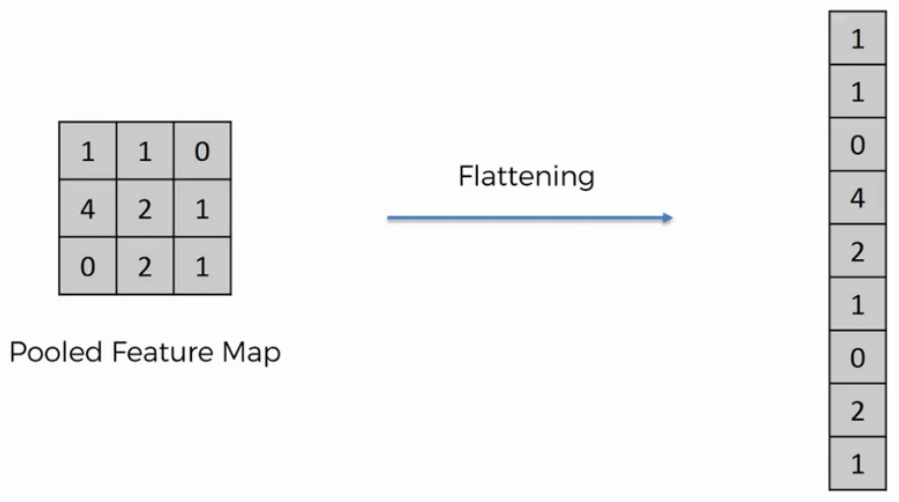

Figure 3: Flattening process. Image taken from Chakrabarty (2019)

The length of the feature vector depends on the dimensions of the image, hence, it is necessary 
to work with images of the same dimensions so the feature vectors have the same size. Since the dimensions of an image must be sufficiently large to represent a distinguishable object, high dimensional feature spaces are generated. Feeding the algorithms with long feature vectors is computationally expensive and affects the model's performance. Therefore, it is appropriate to implement dimension reduction techniques in order to work with fewer and more representative characteristics. Principal Components Analysis and Non-Negative Matrix Function were considered to perform such task.

\section{Principal Components Analysis (PCA)}

PCA uses linear combinations of the variables in order to summarize them into a smaller set of variables that maximizes the variance between observations. Since multiplying a weight with an arbitrary large number will make the resulting variance arbitrarily large the weights must be normalized. The data matrix is assumed to be mean-centered. This is a regular linear algebra problem where the weight that maximizes the variance corresponds to the first eigenvalue (the largest) of the correlation matrix.

\section{Non-Negative Matrix Factorization (NMF)}

This method is based on the idea that given a non-negative matrix of $n x m$ dimensions $M$, two matrices $W$ and $H$ of dimensions $n x r$ and $r x m$, respectively, can be found such that $H=W H$. In order to view $W H$ as a compressed form of the data in $V$ the $r$ is typically chosen such that $(n$ $+m) r<n m$ (Tsuge et al., 2001). Then, the problem can be redefined as $v \approx W h$ where $v$ and $h$ are column of $V$ and $H$. Since $v$ vectors are expressed as a linear combination of $W$ with $h, W$ can be regarded as the basis of the optimized linear approximation of the columns in $V$. $W$ has to allow that the basis vectors discover structure latent in data in order to generate proper aproximations (Tsuge et al. 2001). The fact that the NMF does not accept negative entries leads to a parts-based representation of a structure, since it can be regarded as combining parts to form a whole. Finally, it is important to highlight that the NMF is a widely used technique within image processing. Since images are all represented by non-negative matrices this method generates highly competent outputs for the field problems.

To conclude, it is important to highlight the drawbacks of this approach. When working with pattern recognition problems it is desirable to work with techniques that are invariant to scale, shift, and rotation. That a feature extraction method posses these properties guaranties that the feature vector accurately represents the main characteristics of the image despite of how the object in question appears. In other words, it is not important how the picture of the waste is taken, the position, the angle, and the size of the trash in the image can be neglected, and consequently, the feature vectors of different images of the same type of waste will be quite similar.

Feature vectors composed of a flattened image does not possess these properties, therefore, the

classification algorithms will encounter complication when facing with these situations. Applying certain dimension reduction techniques may lessen the magnitude of the problem, depending on the properties of the chosen method, however, it does not eliminates the defect of the approach. 


\subsubsection{Bag of Features (BoF)}

The main idea of this technique is to work with an order less collection of image characteristics. The bag is formed extracting the features of every image and afterwards construing a "visual dictionary" with the use of a clustering method. Each cluster represents a "term". Then, the features of each image can be mapped to the closest terms in the vocabulary. Finally, the count of how many times a term appears in the picture is extracted and a histogram is generated and is used as feature vector. The process is clearly described in the figure below:

\section{Build Visual Vocabulary}

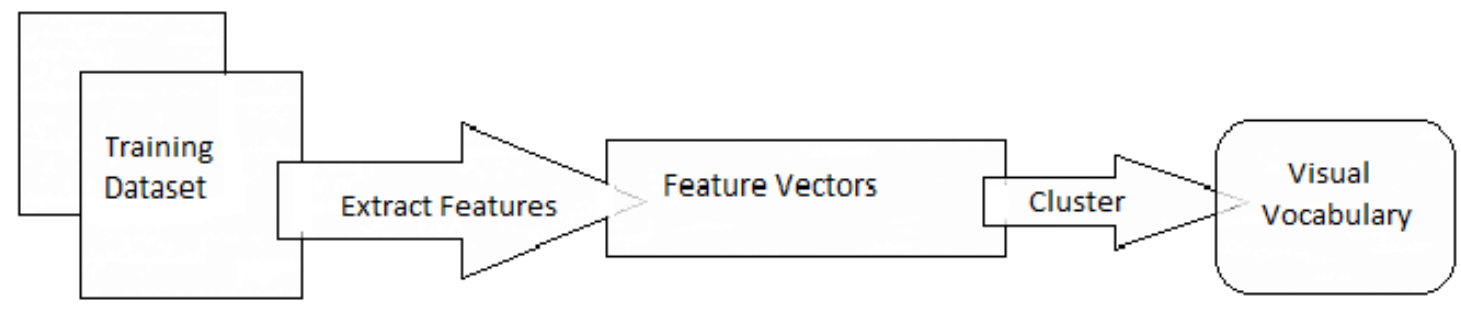

\section{Assign Terms and 3. Generate Term Vector (Image Encoding Vector)}

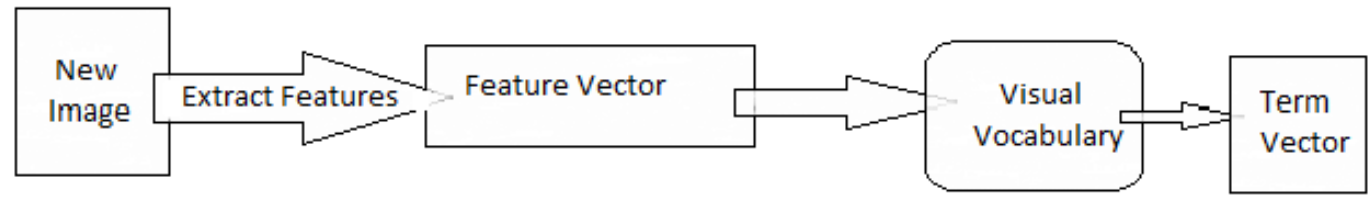

Figure 4: Bag of Features (BoF) representation process. Image taken from Loussaief \& Abdelkrim (2018)

Ultimately, the method attempts to collect local features in order to accurately represent the image from a global perspective. Feature extraction techniques play an important role in this method, and naturally, a wide set of techniques has been developed. A simple but accurate feature extraction method is to run a kernel through the matrix and store the flattened pixel values of the mask in order to construct the dictionary. This method is commonly linked with the Bag of Feature technique. Shift Invariant Feature Transform (SIFT) and Speed Up Robust Features (SURF) algorithms correspond to the current state of art methods and are commonly linked with the Bag of Visual Words technique.

\subsection{Classification Algorithms}

\subsubsection{Logistic Regression}

It uses maximum likelihood estimation after transforming the explained variable into a logit variable. The Logistic Regression not only has the ability to predict the probability of belonging to a class but also to perform classification through the logistic function. The latter is related to the probability of the outcome of the event. The objective function is defined in terms of the maximum likelihood estimation and it can be noted as it follows: 


$$
l(\beta, Y, X)=\sum\left(y_{i} x_{i} \beta-\ln \left(1+\exp x_{i} \beta\right)\right)
$$

The $X$ and $Y$ correspond to the descriptor matrix and the label vector respectively. The $\beta$ corresponds to a vector of weights assigned to each descriptor. The weights are chosen in order to satisfy the maximum likelihood estimation problem and will the value of each weight will indicate the significance of each descriptor.

\subsubsection{K Nearest Neighbors}

K Nearest Neighbors is a non-parametric method that calculates distances between observations in order to perform a prediction. The main idea behind the method is that the feature space of the unlabeled image is taken and then the $k$ nearest feature vectors are found. Afterwards, the label will be assigned by the majority vote scheme. It is important to note that in binary classification the $k$ must be odd in order to avoid ties. One of the main drawbacks of the method is that it treats every feature equally, which may end contributing to image mismatches, specially when it exist a small subset of significant features. The output of the algorithm may vary depending on the type of distance that is decided to use. Some of the most used distances are the Manhattan distance, the Euclidean distance and the Minkowski distance.

\subsubsection{Support Vector Machine}

The SVM was initially proposed for binary classification. The main idea behind the method is to find the optimal hyper plane that maximizes the separation between the two classes. The nearest data samples to the optimal hyper plane are called the support vectors. Let $\left(x_{i}, L_{i}\right)$ with $i=1, \ldots, N$ the data sample were $x_{i}$ is the feature space and $L_{i}$ is the label of the image such that $L_{i} \epsilon\{-1,1\}$. Then a new image feature vector is classified according to the following formula:

$$
f\left(x_{\text {test }}\right)=\sum\left(\alpha_{i} L_{i}\left(\mathbf{x}^{\top} x_{\text {test }}\right)+b\right)
$$

The $\alpha_{i}$ correspond to the non-zero coefficients that solve the quadratic programming problem. The sign of the output determines the class to which the image belongs and is assumed that it is a linearly separable. The algorithm is often used in high complexity problems and has the ability of providing useful information to perform analysis. The SVM uses complex kernels such as the Radial Bias Function $(\mathrm{RBF})$, the polynomial classifier or the sigmoid function and still is easier to analyze in a high dimensional space than other methods. All the calculations take place in a high dimensional space. Support Vector Machines are quite popular due to the variety of kernels that are available for different types of data.

\subsection{Model Evaluation}

The algorithms' performance is going to be evaluated using the accuracy and AUC score as main metrics, accompanied by the corresponding ROC curves. Learning curves are going to be plotted in each test in order to diagnose problems with the generalization ability of the models. The later are going to be computed using the accuracy as reference metric, since AUC cannot be fully analyzed without the ROC curves. 


\subsubsection{AUC and ROC Curves}

The Receiver Operating Characteristic (ROC) Curve contrast two different classification metrics and summarizes them into one plot. The first metric is the sensitivity or true positive rate (TPR), and it is located at the $y$ axis of the ROC curve. The TPR measures the proportion of the postive class that were correctly classified. The second metric is the False Positive Rate (FPR), the complement of the specificity. The FPR measures the proportion of incorrectly classified observations of the negative class. Sensitivity and specificity are two very important metrics in classification problems, thus, the summary of both metrics into one single plot contains a lot of information about the classification performance.

Classification algorithms often generate an output based on an observation's belonging probability to one category. The label is chosen according a threshold, hence, the models performance will vary as the threshold varies. The ROC curve is built upon the variation of this threshold by calculating the corresponding sensitivity and specificity values for each iteration. This allows to represent the overall algorithm performance in a single graph.

What it is expected to see is that the model is capable of obtaining a high sensitivity score while maintaining a high specificity. This is shown when the curve maintains a high TPR for low FPR values. In other words, the closer that the curve gets to the top left corner of the plot, the best that the classifier performs. The Area Under the Curve (AUC) is a summary of the ROC curve, as it name states it indicates the area under the ROC curve. This allows compare models' performance without directly looking at the ROC curve. These are the reasons why the ROC curve and the AUC are considered in this research.

\subsubsection{Learning Curve}

Is a widely used diagnostic tool in machine learning that evaluates the algorithms learning rate. Learning curves are used to determine problems with the learning, such as under fitting or over fitting, as well as whether the models performance can be improved with more data or if the learning process stationed. Moreover, determining if the learning process stationed is quite useful since it reveals that further transformations need to be applied to the data or that a more complex model needs to be implemented.

\section{Results}

The experiments were divided in the two feature extraction techniques mentioned in the previous section. Some image processing techniques were applied in order to enhance the models performance. PCA and NMF were applied to the feature space and the results were contrasted in order to determine which method generates better results. Tests were conducted using Logistic Regression, K Nearest Neighbors, and Support Vector Machine as classification algorithms. The performance of the algorithms was evaluated using 5-fold cross validation using the AUC and accuracy scores as reference metrics. Furthermore, ROC and learning curves where used as a guideline to evaluate the algorithms capability of classification and generalization. A hyper parameter tuning routine was implemented in order to further enhance model performance over the different data setes, however, 
they are not going to be listed in the project due to the length of it.

Experimentation was conducted in a Jupyter Notebook environment using python. Google Colab was used as main platform to perform the operations, image repositories and experiment results were stored in Google Drive. Everything was backed up in a public GitHub repository, for further understanding of the code and experimentation it can be accessed from here. The remaining part of this section will describe the architecture that was adopted in order to perform the experiments and will expose a compilation of the obtained results.

The first stage of the experimentation focused on determining which combination of techniques and algorithms generated the better results when using image pixels as feature vectors. The experimentation contrasted the performance of the methods when the images possessed four different dimensions (10x10, 30x30, 50x50, and 100x100) and were applied a different set of basic processing techniques: converting the image into gray scale, re scaling pixel values, and extracting the borders using the Canny algorithm. Moreover, the feature space dimensions were reduced by applying PCA and NMF. The implemented algorithms for this stage of the experimentation were the Logistic Regression and K Nearest Neighbors. The models were tested with four different data sets: the non-modified feature space (Regular), a PCA feature space (PCA), the principal components that sum the $80 \%$ of the variance of the feature space (PCA80), and a NMF feature space (NMF). The hyper parameters of the algorithms were tuned for each data set and generalization capabilities were evaluated using their learning curve.

The experimentation showed that the two data sets that generated the best results across the experiments were the PCA80 and the NMF. Moreover, this data sets provided the algorithms with a superior generalization ability. The algorithms learning curve showed that whenever the dimensions of an image were increased from 10x10 and they were fed by the Regular or PCA data set the algorithm did not comprehend the relationships of the data, producing that the algorithm under-fitted the data. On the contrary, PCA80 and NMF data sets allowed to generalize appropriately in every case but one. It was observed that the Logistic Regression algorithm lacked the ability of interpreting the relationships among the variables when the edge filters were applied to the data. Whilst, the KNN not only was able of properly fitting the data, but it generated the highest scores of the stage as well. Finally, it was determined that image dimensions are negligible when they are 30x30 or larger since working with the widest feature spaces did not generated good results and that the PCA 80 and NMF scores stationed after 30x30 dimensions. The best results achieved in this stage are listed bellow:

\begin{tabular}{|c|c|c|c|c|}
\hline Models & PCA80 Accuracy Score & PCA80 AUC Score & NMF Accuracy Score & NMF AUC Score \\
\hline LR & 0.71 & 0.76 & 0.72 & 0.76 \\
\hline KNN & 0.71 & 0.85 & 0.70 & 0.77 \\
\hline
\end{tabular}

Table 1: Algorithms best results of stage one experimentation.

In the second stage of the experimentation the Bag of Features approach was taken into account using an 8x8 kernel with a step of three pixels. In addition, the images were segmented in order to reduce the noise of the picture and that the waste occupies a larger space of the image. The 
experiments were conducted with the same methodology of the previous stage with the exception that image dimensions are no longer tested. After the segmentation it was decided to reshape the images so they all possess $75 \times 75$ dimensions. This values were chosen in order to benefit the computational efficiency without compromising the models' performance. The Logistic Regression, K Nearest Neighbors, and the Support Vector Machines where chosen to perform the classification. The results are going to be contrasted by mixing the different image processing techniques listed before and using different types of data sets. PCA80 and NMF image pixel feature vector are still considered for the experimentation. Similarly, four data sets are built from the BoF approach, they are going label them as it follows: BoF-Regular, BoF-PCA, BoF-PCA80, and BoF-NMF. Hyper parameters are tuned for each data set and generalization ability is evaluated.

The BoF approach demonstrated to generate better overall results than working with image pixels. The Logistic Regression accuracy and AUC scores incremented considerably in comparison with the ones generated in the first stage of the experimentation. The learning curves showed that the considered feature space contained sufficiently simple relationships in order for the algorithm to comprehend the data, hence, produce better results. In addition, eliminating the noise in the picture enabled the latter to comprehend images with their edged filtered when working with pixel based feature vectors that are passed through the NMF method, whilst working with PCA80 learning ability remained the same. Moreover, re scaling pixel values of gray scale images generated the worst results of the stage. Performing this transformation to the pixel values affected the BoF ability of creating the vocabulary dictionary.

Furthermore, the K Nearest Neighbors algorithm showed a significant improvement in the accuracy scores generated. Such increment is attributed to the object centering applied since the algorithm performed better even when it worked with PCA80 and NMF data sets. On the other hand, AUC scores did not increment in comparison to the ones obtained in the first stage of experimentation. Finally, the Support Vector Machine achieved the highest scores in every test, demonstrating a better performance when it is fed with the BoF-Regular when the images are just centered and converted to gray scale and with the PCA80 and the image is centered and the edges are filtered. The results of the three classification algorithms over the two data sets mentioned before are listed in the table bellow:

\begin{tabular}{|c|c|c|c|c|}
\hline Models & BoF-Regular Accuracy Score & BoF-Regular AUC Score & PCA80 Accuracy Score & PCA80 AUC Score \\
\hline LR & 0.78 & 0.85 & 0.67 & 0.65 \\
\hline KNN & 0.77 & 0.85 & 0.72 & 0.82 \\
\hline SVM & 0.81 & 0.90 & 0.84 & 0.90 \\
\hline
\end{tabular}

Table 2: Algorithms best results of stage two experimentation.

\section{Conclusions and future research}

The research composes of two main stages of experimentation in which the objective is to determine what are the best approaches to the waste classification problem. The first set of experiments illustrated the importance of dimension reduction techniques when the feature vector is composed of image pixels. Not only it guarantees to increment the algorithms performance but it prevents the model from under fitting the data. In addition, the Logistic Regression is not able of working with 
overly complex sets of data, such as the feature vector generated from edged filtered images. Thus, the necessity of implementing algorithms with a higher capacity of abstraction or to perform image processing techniques that reduces noise is contemplated.

Moreover, second stage experiments demonstrated the superiority of the BoF approach for feature extraction. Describing images local features from a global perspective allowed the algorithms to achieve higher overall performance and it provided sufficiently simple data relations in order to guarantee proper generalization abilities, regardless of the complexity of the data. Nonetheless, object centering provided models with higher abstraction capacity with the ability of achieving outstanding results, as it happened with the Support Vector Machine with the PCA80 data set of edged filtered images.

Future phases of the research will focus on the implementation of modern classification algorithms such as Neural Networks and transfer learning in order to develop a research around the full scope of the waste classification problem. It is expected to work with significantly larger data sets and to perform multi-class classification, hence, building computationally efficient programs is within the objectives of future projects.

\section{References}

Abdel-Shafy, Hussein, \& Mansour, Mona. 2018. Solid waste issue: Sources, composition, disposal, recycling, and valorization. Egyptian Journal of Petroleum, 27(12), 1275-1290.

Aguilar-Virgen, Quetzalli, Vega, Carolina, Taboada-González, Paul, \& Aguilar, Xochitl. 2010. Potential Recovery of Domestic Solid Waste Disposed of in A Landfill. Revista de Ingeniería, $11,16-27$.

Andesco. 2020. El 78\% de los hogares colombianos no recicla. Andesco. Accessed: 2021-02-05.

Arebey, Maher, Hannan, M. A., Begum, Rawshan, \& Basri, Hassan. 2011 (11). CBIR for an Automated Solid Waste Bin Level Detection System Using GLCM.

Aslan, Muhammet, Durdu, Akif, \& Sabanci, Kadir. 2020. Human action recognition with bag of visual words using different machine learning methods and hyperparameter optimization. Neural Computing and Applications, 32(06), 8585-8597.

Camlica, Zehra, Tizhoosh, Hamid, \& Khalvati, Farzad. 2015 (12). Medical Image Classification via SVM using LBP Features from Saliency-Based Folded Data.

Chakrabarty, Navoneel. 2019 (03). A Novel Strategy for Gender Identification from Hand Dorsal Images using Computer Vision.

Chamas, Ali, Moon, Hyunjin, Zheng, Jiajia, Qiu, Yang, Tabassum, Tarnuma, Jang, Jun Hee, Abu-Omar, Mahdi, Scott, Susannah, \& Suh, Sangwon. 2020. Degradation Rates of Plastics in the Environment. ACS Sustainable Chemistry Engineering, XXXX(02).

Geyer, Roland, Jambeck, Jenna, \& Law, Kara. 2017. Production, use, and fate of all plastics ever made. Science Advances, 3(07), e1700782. 
Hannan, M. A., Arebey, Maher, Begum, Rawshan, \& Basri, Hassan. 2012. An automated solid waste bin level detection system using a gray level aura matrix. Waste management (New York, N.Y.), 32(06).

Kumar, Gaurav, \& Bhatia, Pradeep. 2014 (02). A Detailed Review of Feature Extraction in Image Processing Systems.

Liu, Peng, Choo, Kim-Kwang Raymond, Wang, Lizhe, \& Huang, Fang. 2017. SVM or deep learning? A comparative study on remote sensing image classification. Soft Computing, 21(12).

Liu, Yijian, Fung, King-Chi, Ding, Wenqian, Guo, Hongfei, Qu, Ting, \& Xiao, Cong. 2018. Novel Smart Wgaste Sorting System based on Image Processing Algorithms: SURF-BoW and Multi-class SVM. Computer and Information Science, 11(06), 35.

Loussaief, Sehla, \& Abdelkrim, Afef. 2018 (03). Deep learning vs. bag of features in machine learning for image classification.

Mittal, Gaurav, Yagnik, Kaushal, Garg, Mohit, \& Krishnan, Narayanan. 2016 (09). SpotGarbage: smartphone app to detect garbage using deep learning.

Mohajerani, Abbas, Vajna, John, Cheung, Tsz, Kurmus, Halenur, \& Arulrajah, Arul. 2017. Practical recycling applications of crushed waste glass in construction materials: A review. Construction and Building Materials, 156(12), 443-467.

Ozkan, Kemal, Ergin, Semih, Işık, Şahin, \& Işıklı, Idil. 2014. A new classification scheme of plastic wastes based upon recycling labels. Waste management (New York, N.Y.), 35(10).

Sakr, George, Mokbel, Maria, Darwich, Ahmad, Khneisser, Mia, \& Hadi, Ali. 2016 (11). Comparing Deep Learning And Support Vector Machines for Autonomous Waste Sorting.

Satvilkar, Mandar. 2018. Image Based Trash Classification using Machine Learning Algorithms for Recyclability Status.

Semana, Revista. 2020. Así cambiará el reciclaje en Colombia tras entrada en vigencia de una norma. Revista Semana. Accessed: 2021-02-05.

Tomar, Divya, \& Agarwal, Sonali. 2015. A comparison on Multi-class Classification methods based on Least Squares Twin Support Vector Machine. Knowledge-Based Systems, 81(02).

Toğaçar, Mesut, Ergen, Burhan, \& Cömert, Zafer. 2019. Waste Classification using AutoEncoder Network with Integrated Feature Selection Method in Convolutional Neural Network Models. Measurement, 153(12), 107459.

Tsuge, S., Shishibori, M., Kuroiwa, Shingo, \& Kita, K. 2001 (02). Dimensionality reduction using non-negative matrix factorization for information retrieval. vol. 2. 